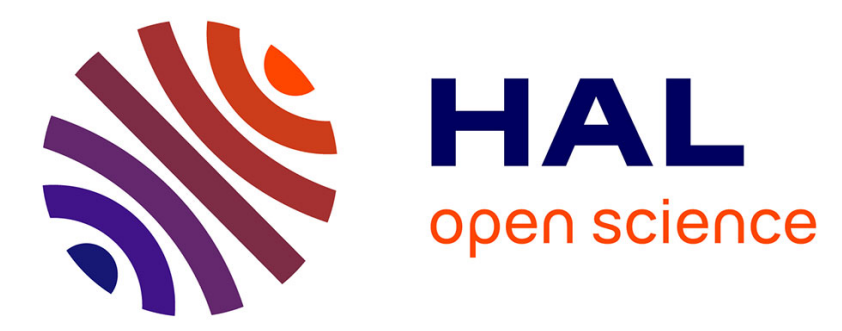

\title{
Preliminary design of a multi-touch ultrasonic tactile stimulator
}

Sofiane Ghenna, Frédéric Giraud, Christophe Giraud-Audine, Michel Amberg, Betty Lemaire-Semail

\section{- To cite this version:}

Sofiane Ghenna, Frédéric Giraud, Christophe Giraud-Audine, Michel Amberg, Betty Lemaire-Semail. Preliminary design of a multi-touch ultrasonic tactile stimulator. World Haptics Conference (WHC), 2015 IEEE, Jun 2015, Chicago, United States. 10.1109/WHC.2015.7177687 . hal-01238296

\section{HAL Id: hal-01238296 \\ https://inria.hal.science/hal-01238296}

Submitted on 4 Dec 2015

HAL is a multi-disciplinary open access archive for the deposit and dissemination of scientific research documents, whether they are published or not. The documents may come from teaching and research institutions in France or abroad, or from public or private research centers.
L'archive ouverte pluridisciplinaire HAL, est destinée au dépôt et à la diffusion de documents scientifiques de niveau recherche, publiés ou non, émanant des établissements d'enseignement et de recherche français ou étrangers, des laboratoires publics ou privés. 


\title{
Preliminary design of a multi-touch ultrasonic tactile stimulator
}

\author{
Sofiane GHENNA ${ }^{1}$, Frederic GIRAUD ${ }^{1}$, Christophe GIRAUD-AUDINE ${ }^{1,2}$, \\ Michel Amberg ${ }^{1}$ and Betty LEMAIRE-SEMAIL ${ }^{1}$
}

\begin{abstract}
This paper presents a method to control ultrasonic waves on a beam, allowing to obtain a Multi-touch ultrasonic tactile stimulation in two points, to give the sensation to two fingers, from two piezoelectric transducers. The multi-modal approach and the vector control method are used to regulate the vibration amplitude, in order to modulate the friction coefficient with the fingers. An analytical modelling is presented, with experimental validation. Finally a psychophysical experiment shows that a multi-touch ultrasonic tactile stimulation is possible.
\end{abstract}

Keywords: Multi-touch, Haptics, Tactile stimulation, Multi-modal approach, Ultrasonic waves.

\section{INTRODUCTION}

Nowadays, the sense of touch plays an important role for the recognition, discovery and use of objects around us. This attracted the interest of researchers to implement a simulative approach similar to vision or hearing, and to recreate the touch sensation. Some modern devices are able to reproduce the illusion of the textured surfaces under the finger [1], [2]. Some techniques are based on friction control between a surface and the finger. They involve ultrasonic vibrations [3] [4], [5], or electro-vibration [6], [7]. For the first, a squeeze film effect is used to modulate the friction coefficient, by creating an air film between the user's finger and the surface. For the second electrostatic forces are employed to attract the finger toward the physical surface. The provided stimulation is uniform under the fingertip, so they are categorized as global stimulation. To give the illusion of textured surfaces, these devices modulate the stimulation amplitude, according to the position of the finger. The main issue of these techniques is that the sensation are identical all over the touched area. Hence, it is not possible to control the sensation on more than one finger, in spite of the temporal modulation [8].

There exist however devices with localised tactile stimulation. For example, [9] focuses the vibrations in one or more points of a plate, using time-reversal of flexural waves. It should be noted that this method requires a learning step, and

1 S. GHENNA, F. GIRAUD, M. AMBERG, B. LEMAIRESEMAIL are with Laboratoire d'Electrotechnique et d'Electronique de Puissance, 59000 Villeneuve d'Ascq, France e-mail: sofiane.ghennaded.univ-lillel.fr, Frederic.Giraudeuniv-lillel.fr,

Michel.Amberg@univ-lillel.fr, Betty.Semailapolytech-lille.fr,

2 C. GIRAUD-AUDINE is with Ecole Nationale Suprieure d'Arts et Mtiers Paris-Tech, Boulevard Louis XIV, 59000 Lille, France Christophe.Giraud-Audinedircica.univ-lillel.fr a large number of piezoelectric actuators. For the electrovibration, the authors in [10] proposed an approach based on detecting position, direction and force for multi-finger electrostatic haptic system, through a stimulator pad. By employing multiple stimulator pads and applying different voltages to different stimulators, it provides independent stimulus to several fingers. However, the system requires a multiple stimulator pads attached to the user's fingers for generating force feedback, and is limited to the speed of the stimulation pads. Others techniques such as matrix interface [11], [12] are based on the generation of a $3 \mathrm{D}$ surface, allowing the use of one or more fingers, which may vary in both space and time. So the resolution is related to the number of used actuators.

Currently there are no solutions able to provide a multitouch tactile stimulation based on the squeeze film effect. The main objective of this paper is to present a control method, allowing to have a differentiated tactile stimulation, using a squeeze film effect to obtain different sensations for the use of two fingers simultaneously.

In the second section we propose the principle of the applied method. The excitation conditions are presented in the third section. Finally the result of this study is illustrated with an experimental validation in the fourth part.

\section{PRINCIPLE}

\section{A. Limitations of current devices}

In squeeze film effect one flexion vibration mode at a resonance frequency above $20 \mathrm{kHz}$ is used [2]. This standing wave is a function of the location $x$ and time $t$, with vibration amplitude named $W$. Then the vibration at any point of the beam is given by:

$$
w(x, t)=W \sin (\omega t) \phi(x)
$$

where $\phi(x)$ denote the spatial mode shape function, and $\omega$ is the angular frequency. The vibration amplitude at position $x_{i}$ of this standing wave is determined by a single and dependant variable $W$. Hence, there is no possibility to control areas with different vibrations amplitude, in order to get predefined friction coefficients $\mu$ under different fingers. And by using one vibration mode, the vibration amplitude being zero at the nodal lines as described in [13]. However, it is known that a mechanical structure responds to the excitations with infinite modes. This property will be used to obtain the required vibration shape of multi-touch interaction. In the following, we are interested in the simplest case of a beam. 


\section{B. Forced vibration in finite beam}

Considering a thin beam, with rectangular cross section denoted by $A$, length by $L . \rho, E$ are respectively density and modulus of elasticity, $I$ is the moment inertia of the cross section. The transverse vibration of a uniform elastic homogeneous isotropic Euler-Bernoulli beam [14] can be written in Cartesian coordinates as

$$
E I \frac{\delta^{4} w}{x^{4}}+\rho A \frac{\delta^{2} w}{\delta t^{2}}+r_{a} \frac{\delta w}{\delta t}=p(x, t)
$$

with: $w=w(x, t)$ the deflection of the beam at point $x$ and time $t, \delta$ is the partial-derivative, and $p(x, t)$ denotes the load per unit length of the beam at point $x$ and time $t$, while $r_{a}$ represents the coefficient of external damping of the beam. It is possible to determine the deformation mode shapes, and the frequency spectrum of the beam, by using the analytical model of Euler-Bernoulli eq.2 developed in [15]. For a free-free boundary conditions, the first four mode shapes are represented in Fig 1. This analytical modelling



Fig. 1. First 4 mode shapes for a free-free beam

shows that the solution of wave propagation in a finite beam has solutions for special frequency values. In this paper, harmonic vibrations are only considered enabling the use of the complex notation so that: $\underline{w}$, with $w$ is the real part of $\underline{w}$, and $\underline{w}=\underline{W} e^{j \omega t}$, where $\underline{W}=W e^{j \alpha}, \alpha$ and $W$ are respectively the argument and the magnitude of $\underline{W}$. If we take into account the contribution of every vibration mode excited in the beam, then the variation can be written as:

$$
\underline{w}(x, t)=\sum_{n=1}^{\infty} \underline{W_{n}} \phi_{n}(x) e^{j \omega t}
$$

Where $\underline{\mathrm{w}}$ includes real and imaginary part of the vibration, at location $x$, and time $t, \omega$ is the angular frequency of the flexural wave, and $j=\sqrt{-1}$.

$\phi_{n}(x)$ is the deformation mode shapes of the $n^{\text {th }}$ mode, and is fixed for the beam.

$W_{n}$ represents the modal amplitude of the $n^{\text {th }}$ mode. We suppose that we can control each mode independently. Hence, the deformation $\underline{w}(x, t)$ depends theoretically on an infinite degrees of freedom which are $W_{n}$. This property is used in the case of multi-touch interaction.

\section{Multi-touch ultrasonic tactile stimulation}

For a multi-touch ultrasonic tactile stimulation the vibrations, $w\left(x_{i}, t\right)$ must be imposed independently to obtain a different friction coefficients $\mu_{i}$ predefined at several positions $x_{i}$, where $i$ is an integer.

In this paper, we limit the study to two flexion modes to generate the deformation of a beam, allowing the use of two fingers. From eq.3, considering two vibration modes denoted $\mathrm{A}$ and $\mathrm{B}$, we have:

$$
\underline{W}(x)=\underline{W}_{A} \phi_{A}(x)+\underline{W}_{B} \phi_{B}(x)
$$

From the combination of two flexion modes, it is possible to obtain a function $\underline{W}(x)$ which depends on the modal amplitude $\underline{W}_{A}$ and $\underline{W}_{B}$. To obtain this, we need a specific excitation conditions of flexural waves, a multi-modal approach thus is required to realize this.

\section{Multi-modal approach}

The multi-modal approach consists in exciting two successive flexion modes of the beam, around the middle of their resonant frequency as depicted in Fig.2. It has been shown

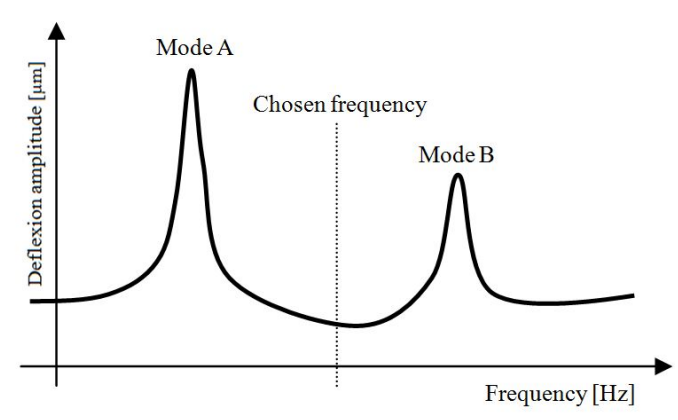

Fig. 2. Excitation between two successive flexion modes

in [5], that the frequency of vibration has no influence on the perceived sensation, above a threshold value of $20 \mathrm{kHz}$. The dimensions of the beam are adjusted in order to have the modes A and B above $20 \mathrm{kHz}$.

Several studies were focused on the generation of ultrasonic waves on a beam, including the transportation of objects by travelling wave [16], [17]. This methodology has been used for the generation of two vibration modes with the same amplitude shifted by $\frac{\pi}{2}$. Here we use this principle in another way to generate a differentiated tactile stimulation as shown in Fig.3. By the combination of two

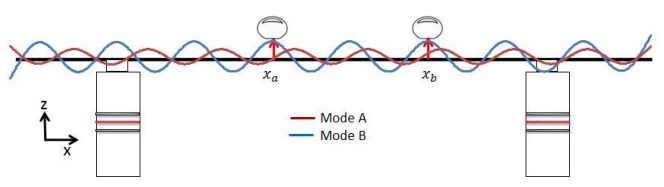

Fig. 3. Generating principle of two successive flexion modes

successive bending modes, ( the red and blue curve represent 
vibration mode $\mathrm{A}$, and mode $\mathrm{B}$ respectively), it is possible to obtain two different vibrations amplitude in two points of the beam, as shown under the two fingers, because each vibration mode has a specific modal amplitude. These vibration amplitudes $\underline{W}\left(x_{a}\right), \underline{W}\left(x_{b}\right)$ depend on both of the deformation mode shapes, and their modal amplitude, which makes two independent variables. Figure 4 shows a beam operated by two piezoelectric actuators located at distance $x_{A}, x_{B}$ from each end of the beam. The blue curve represents the contribution of the two vibration modes in a transverse direction at a given moment, expressed by eq.4, at each position of the beam.

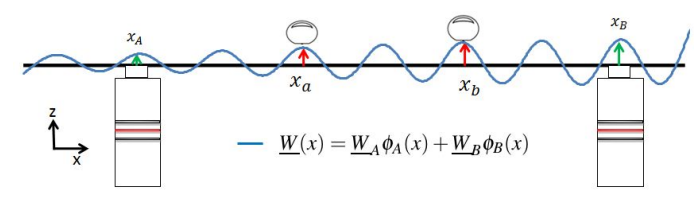

Fig. 4. Generating principle of a differentiated tactile stimulation

If we consider two points of the beam $x_{a}$ and $x_{b}$, the harmonic vibration at these positions is given by:

$$
\begin{aligned}
& \underline{W}\left(x_{a}\right)=\underline{W}_{A} \phi_{A}\left(x_{a}\right)+\underline{W}_{B} \phi_{B}\left(x_{a}\right) \\
& \underline{W}\left(x_{b}\right)=\underline{W}_{A} \phi_{A}\left(x_{b}\right)+\underline{W}_{B} \phi_{B}\left(x_{b}\right)
\end{aligned}
$$

Introducing the matrix notation, eq.5 and eq. 6 become:

$$
\left(\begin{array}{l}
\underline{W}_{x a} \\
\underline{W}_{x b}
\end{array}\right)=\left(\begin{array}{ll}
\phi_{A}\left(x_{a}\right) & \phi_{B}\left(x_{a}\right) \\
\phi_{A}\left(x_{b}\right) & \phi_{B}\left(x_{b}\right)
\end{array}\right)\left(\begin{array}{l}
\underline{W}_{A} \\
\underline{W}_{B}
\end{array}\right)
$$

the two vibration amplitude can be controlled directly by the modal amplitude. Then the control of these modal amplitudes is necessary. Inversely, under the assumption of a non-singular matrix $\phi_{x_{a}, x_{b}}$, the vibration amplitude of mode $\mathrm{A}$ and $\mathrm{B}$ are given by:

$$
\left(\underline{W}_{A}\right)=\phi_{x_{a}, x_{b}}^{-1}\left(\underline{W}_{x_{b}} \underline{\underline{W}}_{a}\right)
$$

Similarly for the vibration amplitude at the position of the actuator $\underline{w}_{x_{A}}$, and $\underline{w}_{x_{B}}$ :

$$
\left(\begin{array}{l}
\underline{W}_{x_{A}} \\
\underline{W}_{x_{B}}
\end{array}\right)=\left(\begin{array}{ll}
\phi_{A}\left(x_{A}\right) & \phi_{B}\left(x_{A}\right) \\
\phi_{A}\left(x_{B}\right) & \phi_{B}\left(x_{B}\right)
\end{array}\right)\left(\begin{array}{l}
\underline{W}_{A} \\
\underline{W}_{B}
\end{array}\right)=\phi_{x_{A}, x_{B}}\left(\underline{W}_{A}\right)
$$

The vibrations of transducers impose the dynamic of the modal amplitudes. Substituting eq. 8 in eq. 9 gives:

$$
\left(\underline{W}_{x_{A}}\right)=\phi_{x_{A}, x_{B}} \cdot \phi_{x_{a}, x_{b}}^{-1}\left(\underline{W}_{x_{b}} x_{a}\right)
$$

eq.10 allows us to calculate the required vibration amplitude of the transducers as a function of the predefined vibration amplitude under each finger. Hence, to generate a differentiated tactile stimulation, it is necessary to know the position of each finger $x_{a}$ and $x_{b}$, the positions of the actuators $x_{A}$ and $x_{B}$, the deformed shape matrix at the position of the actuators $\phi_{x_{A}, x_{B}}$, which is a constant matrix, and the deformed shape matrix $\phi_{x_{a}, x_{b}}$, which depends on the position of the two fingers. By imposing a desired vibration amplitude $\underline{W}_{x_{a}}$ and $\underline{W}_{x_{b}}$ under two fingers, we determine the reference vibration amplitude $\underline{W}_{x_{A}}$ and $\underline{W}_{x_{B}}$, to be imposed on each actuator as described in Fig.5. By this way, it is possible to obtain two

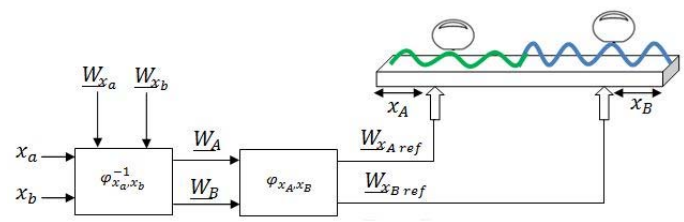

Fig. 5. Control strategy for a Multi-touch ultrasonic tactile stimulation

different friction coefficients at this different positions, this allowing to obtain a multi-touch tactile stimulation.

\section{EXCITATION CONDITIONS OF FLEXURAL WAVE AND TRANSDUCERS}

\section{A. Wavelength}

The wavelength depends on the excitation frequency, and the different physical characteristics of the beam material. However, to differentiate the sensations, it is necessary to have a gap between nodes and anti-nodes. Hence the half wavelength should be larger than width of finger, which is approximately equal $17 \mathrm{~mm}$.

\section{B. Frequency and vibration modes}

The analytical model of Euler-Bernoulli eq. 2 was simulated to determine the frequency spectrum of the beam given in Fig.6, the deformed mode shapes presented in Fig 7. A beam made of aluminium was chosen, because of the excellent acoustical characteristics of this material; its parameters are given in Tab.I. The dimensions of the beam has been chosen in such a way that the half wavelength is $20 \mathrm{~mm}$, and the resonance of the beam is around $28 \mathrm{kHz}$, to obtain the optimal operating conditions of the transducers, and a high vibration amplitude. Then, the contribution of two vibration modes to produce a squeeze-film effect was taken into account.

TABLE I

BEAM'S CHARACTERISTIC

\begin{tabular}{|c|c|}
\hline Young's modulus E & $57 \mathrm{GPa}$ \\
\hline Poisson's ratio $\gamma$ & 0.33 \\
\hline Density $\rho$ & $2561 \mathrm{~kg} / \mathrm{m}^{3}$ \\
\hline Length $\mathrm{L}$ & $350 \mathrm{~mm}$ \\
\hline height $\mathrm{h}$ & $6 \mathrm{~mm}$ \\
\hline width $\mathrm{b}$ & $6 \mathrm{~mm}$ \\
\hline
\end{tabular}




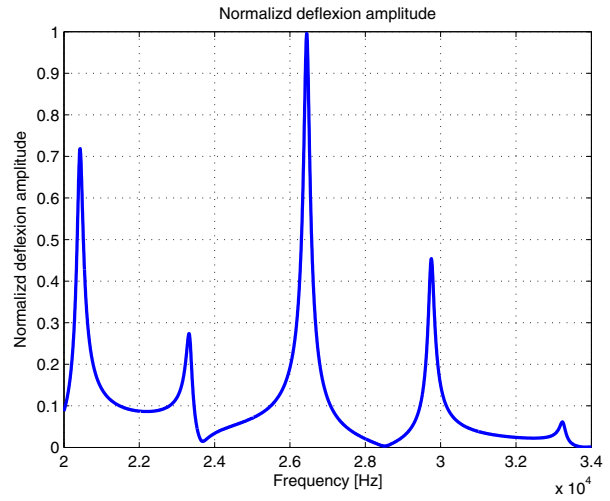

Fig. 6. Normalized deflection amplitude at a given position of the beam

From Fig.6, the choice of the vibration modes was laid on the $16^{\text {th }}$ and $17^{\text {th }}$ mode shape corresponding to $26460 \mathrm{kHz}$ and $29760 \mathrm{kHz}$ which are denoted by mode shape A, and B respectively. It should be noted that there are other vibration modes between two successive flexion modes, as extensional and torsional mode, but only bending mode are excited transversely.

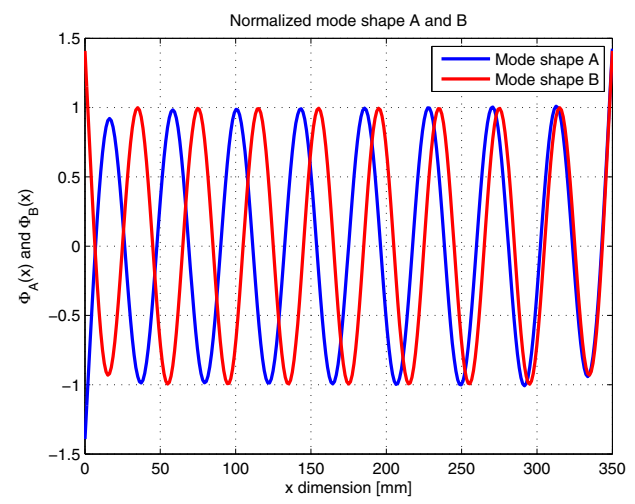

Fig. 7. Simulated normalized mode shape A and B

\section{Position of transducers}

The two piezoelectric actuators are positioned near the anti-nodes of each mode, in order to optimizing the electromechanical couplings. The modal analysis of Fig.7 can be used to determine the position of the transducers on the anti-nodes, or the formula described in [18] can be used.

$$
x_{A}=x_{B}=n \frac{\lambda}{2}+\frac{7}{8}
$$

where $x_{A}, x_{B}$ are the position of the transducers from both ends of the beam, $n$ is an integer, $\lambda$ is the wavelength. The two transducers are placed at $x_{A}=x_{b}=60 \mathrm{~mm}$, at the ends of the beam, with wavelength of $41.5 \mathrm{~mm}$.

\section{Deformation mode shapes matrix $\phi_{x_{a}, x_{b}}$}

Accordingly to eq.8, the condition is to obtain a non singular deformation mode shapes matrix $\phi_{x_{a}, x_{b}}$, Fig8 shows the determinant of $\phi_{x_{a}, x_{b}}$ as a function of $x_{a}$ and $x_{b}$. In this figure the area in green shows that, a dual touch interaction cannot be calculate especially for extremely close distances lower to $1 \mathrm{~mm}$, and at the end of the beam. For the first it is possible to choose another adjacent point, because the finger width is approximatively $8 \mathrm{~mm}$. For the second may be due to the boundary conditions, and for the anti-diagonal zones when the distance between the two fingers are very small, what is physically impossible.

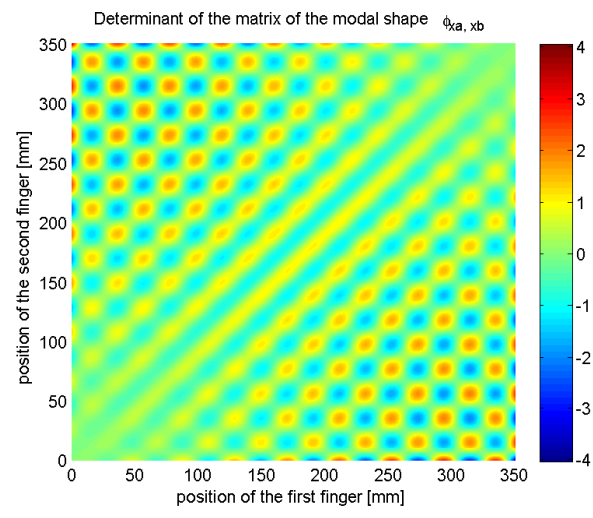

Fig. 8. Determinant of the matrix of the modal shape $\phi_{x a, x b}$

\section{E. Influence of finger pressure}

The transducer is modeled in rotating frame, and the decoupling according to two-axis allows us to obtain a double independent closed loop control. It is possible to control the transducer vibration amplitude and its relative phase, in steady state even in transient by acting on the amplitude of the supply voltage. This allows the transducers to control the vibration amplitude on the surface beam $\underline{W}_{x_{A}}$ and $\underline{W}_{x_{B}}$ in a closed loop. When the finger applied a normal force, this force is rejected as perturbation. That is to say if the vibration amplitude of transducers are imposed in open loop, then when applying a force, this vibration amplitude decrease.

\section{EXPERIMENT VALIDATION}

An aluminium beam is actuated by two Langevin transducers, which were associated with horns. The whole system is fixed on a solid support, which allows to move the beam for measuring the vibration velocity at every point of the beam, using an interferometer (OFV-525/-5000-S) as shown in Fig.9. A graphical user interface is used to control the vibrations amplitude of the two actuators independently, through a DSP (TI 2812) and two amplifiers (HSA 4051).

\section{A. Identification of vibration modes}

A cartography was performed to measure the vibration amplitude of each point in the beam, for the mode shape A and B as shown in Fig.10.

Fig.10 is used to determine the deformation mode shapes $\phi_{x_{A}, x_{B}}$ and $\phi_{x_{a}, x_{b}}$, we obtain: 


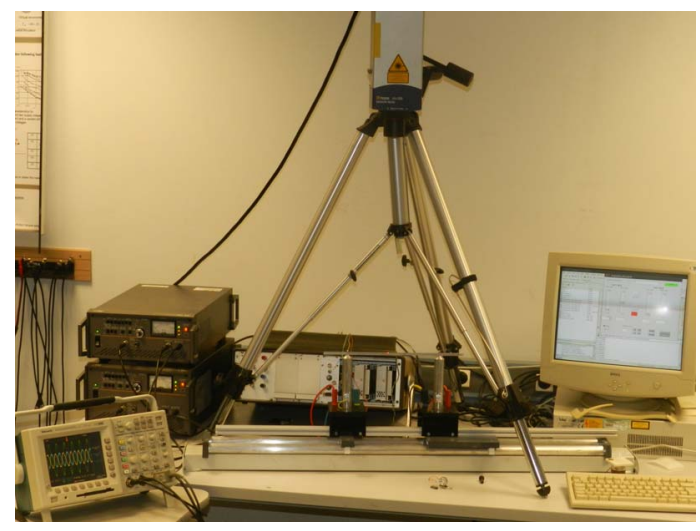

Fig. 9. The experimental setup



(a) Normalized measured mode $\mathrm{A}$ at $26373 \mathrm{~Hz}$

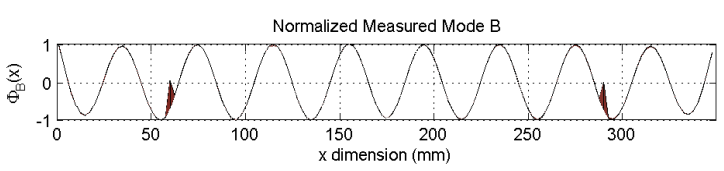

(b) Normalized measured mode B at $29369 \mathrm{~Hz}$

Fig. 10. Normalized measured mode A and B

$$
\left(\begin{array}{ll}
\phi_{A}\left(x_{A}\right) & \phi_{B}\left(x_{A}\right) \\
\phi_{A}\left(x_{B}\right) & \phi_{B}\left(x_{B}\right)
\end{array}\right)=\left(\begin{array}{cc}
-0.9680 & -0.6949 \\
1 & -0.7447
\end{array}\right)
$$

Two points were arbitrarily selected $x_{a}=120 \mathrm{~mm}, x_{b}=$ $250 \mathrm{~mm}$, so that the vibration amplitude at these two points do not represent nodes nor anti-nodes of mode A and B. The matrix of the deformation mode shapes at these positions are given by:

$$
\left(\begin{array}{ll}
\phi_{A}\left(x_{a}\right) & \phi_{B}\left(x_{a}\right) \\
\phi_{A}\left(x_{b}\right) & \phi_{B}\left(x_{b}\right)
\end{array}\right)=\left(\begin{array}{cc}
0.9436 & 0.6809 \\
0.9715 & -0.7388
\end{array}\right)
$$

Now if it is desired to obtain a vibration amplitude under the first finger $0.8 \mu \mathrm{m}$ peak to peak and $0 \mu \mathrm{m}$ under the second finger independently, corresponding to the smooth and the rough area respectively. In this case we determine the vibration amplitude to impose on each actuator using eq. 10 , then:

$$
\left(\begin{array}{l}
\underline{W}_{x_{A}} \\
\underline{W}_{x_{B}}
\end{array}\right)=\left(\begin{array}{c}
-0.4093 \\
0.0045
\end{array}\right) \mu \mathrm{m}
$$

In Fig 11 it is shown the vibrations amplitude at two different points for $x_{a}$ and $x_{b}$ as desired under the first finger $W_{x_{a}}$, and the second finger $W_{x_{b}}$, with a supply voltage of $160 \mathrm{~V}$ peak to peak for each transducer. The system is symmetrical between the transducers.

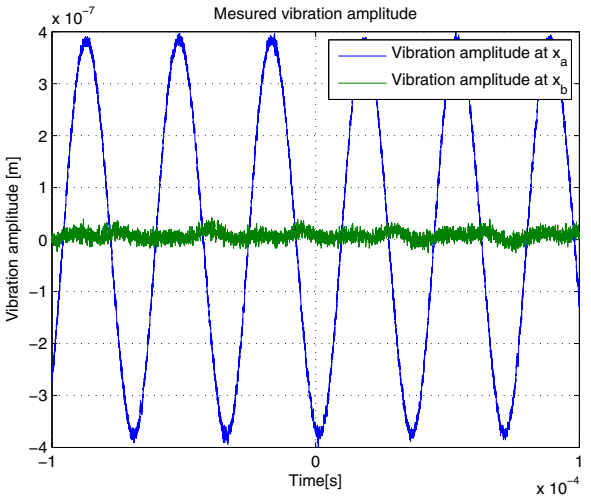

Fig. 11. Measured Vibration amplitude at position $x_{1}=120 \mathrm{~mm}$ and $x_{2}=$ $250 \mathrm{~mm}$

\section{B. Psychophysical validation}

In order to evaluate the ability of the interface to provide a multi friction modulation over the surface, a psychophysical experience has been performed using two-alternatives forcedchoice method described in [19].

It has been asked to 6 participants ( 5 males, 1 female; age range: $22-32$ years) to evaluate simultaneously two different areas of the tactile interface with the index of the right and left hand. Before the exploration it was set in the middle of the right explored areas one anti-node with $1.4 \mu \mathrm{m}$ peak to peak vibration amplitude, and one node in the left area, or inversely. It was asked to the participants to indicate "which of the two areas were the smoothest", corresponding to a smallest friction coefficient. As indicated by the applied method, the participant was forced to choose one side after the free exploration, (even if the participants could not differentiate those two sensations), which were allowed in a surrounding area, $1 \mathrm{~cm}$ large, for 8 seconds. The experience was repeated for 10 times with a randomization of the trials. The participants were able to identify as smoother the area with high vibration amplitude with an average success rate of $78.3 \%$ with a standard variation of 7.5 . The hypothesis test against the random answer was refused with a $p<0.1$ leading to accept the conclusion that a multi-touch stimulation was performed.

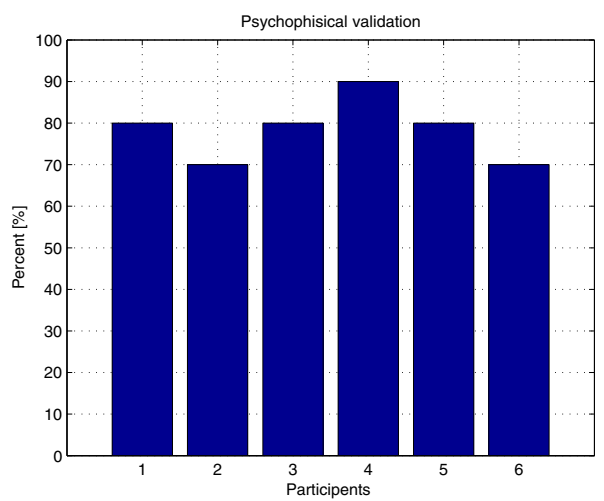

Fig. 12. Results of psychophysical testing 


\section{Discussion}

The possibility to obtain simultaneous different sensations on different sections of the beam has been proved. The performed stimulation was static, but nevertheless the difference in friction was perceivable by the users with significance. The distance between the nodes is $2 \mathrm{~cm}$, which is comparable with the finger contact area for a light touch exploration [20]. An half wavelength above $20 \mathrm{~mm}$ can be implemented to obtain a larger uniform explorable area at the cost of thicker beam. In future works a larger half wavelength will be employed to obtain a bigger difference in absolute friction due to the better uniformity of the vibrations under the fingertip. Moreover, a tracking system for the fingers [8] will be implemented to introduce a position based multi-touch stimulation to render different textures on different fingers. This tactile stimulator was produced in a thin beam, using its $16^{\text {th }}$ and $17^{\text {th }}$ vibration modes, but this method can be employed only with harmonic vibrations.

\section{A. Limitations of the proposed approach}

The proposed concept can be extended to 5 fingers for example, ie 5 positions by taking into account 5 vibration modes, then 5 actuators are required to generate 5 vibration amplitudes, accordingly to eq.7. Theoretically only flexion modes are excited. However, in practical, there is one problem to get these successive flexion modes. The banding modes, unfortunately, cannot be excited independently without affecting the other modes. Therefore, the modal analysis of plates will show that other types of vibration modes are existed and these conditions can highly influence the results. Thus the use of the beam is appropriate for this approach (2 fingers). The effect of actuator location can be resumed in eq. 9 for example if these location presents nodes then no vibration modes are excited, because the deformation modes shape at these positions is equal to zero. The relation between the frequency of the modes and the operating frequency of the transducers is important; the optimum is to obtain two vibration modes equally distant from the resonance of the actuators.

\section{CONCLUSION}

In this paper it is presented a new method to control the vibration amplitude on a finite beam in a closed loop control. And to produce a multi-touch tactile stimulation in two points of the beam independently, even in presence of perturbations, from the exploitation of two vibration modes. That is to say two vibration modes for two fingers, several fingers for several vibration modes, allowing to obtain a multi-finger tactile feedback, using a multi-modal approach.

\section{ACKNOWLEDGMENT}

This work has been carried out within the framework of the project StimTac of IRCICA (institut dde recherche sur les composants logiciels et matériel pour la communication avancé), and the Project Mint of Inria.

\section{REFERENCES}

[1] M. Biet, F. Giraud, and B. Lemaire-Semail, Squeeze film effect for the design of an ultrasonic tactile plate, IEEE Transactions on Ultrasonics, Ferroelectrics, and Frequency Control, vol. 54, no. 12, pp. 26782688, Dec. 2007.

[2] F. Giraud, M. Amberg, B. Lemaire-Semail, and G. Casiez, Design of a transparent tactile stimulator, in 2012 IEEE Haptics Symposium (HAPTICS), 2012, pp. 485489.

[3] D. J. Meyer, M. A. Peshkin, and J. E. Colgate, Fingertip friction modulation due to electrostatic attraction, in World Haptics Conference (WHC), 2013, 2013, pp. 4348.

[4] L. Winfield, J. Glassmire, J. E. Colgate, and M. Peshkin, T-PaD: Tactile Pattern Display through Variable Friction Reduction, in EuroHaptics Conference, 2007 and Symposium on Haptic Interfaces for Virtual Environment and Teleoperator Systems. World Haptics 2007. Second Joint, 2007, pp. 421426.

[5] T. Watanabe and S. Fukui, A method for controlling tactile sensation of surface roughness using ultrasonic vibration, in , 1995 IEEE International Conference on Robotics and Automation, 1995. Proceedings, 1995, vol. 1, pp. 11341139 vol.1.

[6] E. Vezzoli, M. Amberg, F. Giraud, and B. Lemaire-Semail, Electrovibration Modeling Analysis, in Haptics: Neuroscience, Devices, Modeling, and Applications, M. Auvray and C. Duriez, Eds. Springer Berlin Heidelberg, 2014, pp. 369376.

[7] R. M. Strong and D. Troxel, An Electrotactile Display, IEEE Transactions on Man-Machine Systems, vol. 11, no. 1, pp. 7279, Mar. 1970.

[8] M. Biet, G. Casiez, F. Giraud, and B. Lemaire-Semail, Discrimination of Virtual Square Gratings by Dynamic Touch on Friction Based Tactile Displays, in symposium on Haptic interfaces for virtual environment and teleoperator systems, haptics 2008, pp. 41-48.

[9] C. Hudin, J. Lozada, and V. Hayward, Localized tactile stimulation by time-reversal of flexural waves: Case study with a thin sheet of glass, in World Haptics Conference (WHC), 2013, 2013, pp. 67-72.

[10] T. Nakamura and A. Yamamoto, Position and Force-direction Detection for Multi-finger Electrostatic Haptic System Using a Vision-based Touch Panel, presented at the ACHI 2014, The Seventh International Conference on Advances in Computer-Human Interactions, 2014, pp. 160-165.

[11] D. Prescher, G. Weber, M. Spindler A Tactile Windowing System for Blind Users, In Proceedings of the 10th international ACM SIGACCESS Conference on Computers and Accessibility (Orlando, Florida, USA, October 25-27, 2010). Assets '10. ACM, New York.

[12] Schiewe, M. Kohlmann, W. Nadig, O. Weber, G.What You Feel is What You Get: Mapping GUIs on Planar Tactile Displays, In Universal Access in HCI, Part II, HCII 2009. LNCS, vol. 5615, pp. 564-573 (2009)

[13] N. D. Marchuk, J. E. Colgate, and M. A. Peshkin, Friction measurements on a Large Area TPaD, in 2010 IEEE Haptics Symposium, 2010, pp. 317320.

[14] K. F. Graff, Wave Motion in Elastic Solids. Courier Corporation, 1975.

[15] M. Abu-Hilal, Forced vibration of Euler-Bernoulli beams by means of dynamic Green functions, Journal of Sound and Vibration, vol. 267, no. 2, pp. 191-207, Oct. 2003.

[16] F. Giraud, C. Giraud-Audine, M. Amberg, and B. Lemaire-Semail, Vector control method applied to a traveling wave in a finite beam, IEEE Transactions on Ultrasonics, Ferroelectrics, and Frequency Control, vol. 61, no. 1, pp. 147-158, Jan. 2014.

[17] B.-G. Loh and P. I. Ro, An object transport system using flexural ultrasonic progressive waves generated by two-mode excitation, IEEE Transactions on Ultrasonics, Ferroelectrics, and Frequency Control, vol. 47, no. 4, pp. 994-999, Jul. 2000.

[18] M. Kuribayashi, S. Ueha, and E. Mori, Excitation conditions of flexural traveling waves for a reversible ultrasonic linear motor, The Journal of the Acoustical Society of America, vol. 77, no. 4, pp. 14311435, Apr. 1985.

[19] L. A. Jones and H. Z. Tan, Application of Psychophysical Techniques to Haptic Research, IEEE Transactions on Haptics, vol. 6, no. 3, pp. 268-284, Jul. 2013.

[20] M. J. Adams, S. A. Johnson, P. Lefvre, V. Lvesque, V. Hayward, T. Andr, and J.-L. Thonnard, Finger pad friction and its role in grip and touch, Journal of The Royal Society Interface, vol. 10, no. 80, p. 20120467, Mar. 2013. 